\title{
Olmesartan Enteropathy
}

\author{
Hugh James Freeman* \\ Department of Medicine (Gastroenterology) University of British Columbia, Vancouver, BC, Canada \\ *Corresponding author: hugfree@shaw.ca
}

\begin{abstract}
Celiac disease is an immune-mediated small intestinal mucosal disorder with characteristic, but nonspecific histopathological changes. A number of heterogeneous disorders may produce similar changes in small bowel biopsies, but these fail to respond to a gluten-free diet. Recently, it has become increasingly appreciated that different medications may cause this sprue-like intestinal disease, including a specific angiotensin II receptor antagonist, olmesartan, an agent often used for treatment of hypertension. This enteropathy appears to occur as an apparently rare adverse event, often presenting with severe diarrhea, malabsorption and weight loss following chronic long-term use of the drug. Serological studies, particularly for tissue transglutaminase IgA antibodies, are negative and biopsies may show moderate to severe changes in mucosal architecture similar to celiac disease. Subepithelial collagen deposits may also occur. Clinical and biopsy changes fail to respond to a strict gluten-free diet, but cessation of olmesartan use appears to result in resolution.
\end{abstract}

Keywords: celiac disease, sprue-like intestinal disease, drug-induced enteropathy, refractory celiac disease, collagenous sprue

Cite This Article: Hugh James Freeman, “Olmesartan Enteropathy.” International Journal of Celiac Disease, vol. 4, no. 1 (2016): 24-26. doi: 10.12691/ijcd-4-1-6.

\section{Introduction}

Sprue-like intestinal disease $[1,2]$ is a clinically distinctive, but likely heterogeneous enteropathic process refractory to different dietary restrictions, including gluten. While some infectious agents should be excluded, other entities may cause similar enteropathic effects, including "new" disorders associated with transplantation, immune deficiency syndromes and enteritis following colon resection for severe colitis. A number of medications have been identified that may cause this sprue-like intestinal mucosal inflammatory process, including alcohol, antibiotics (eg., neomycin), non-steroidal anti-inflammatory agents (eg., sulindac), stathmokinetic and chemotherapeutic drugs (eg.., colchicine, vincristine, methotrexate) and, more recently, immunosuppressants (eg., azathioprine, mycophenolate mofetil and some monoclonal biological agents (eg., ipilimumab) used in management of advanced neoplastic disorders, such as malignant melanoma.

In many of these drug-induced enteropathies, the colonic mucosa may also be involved with a microscopic inflammatory process, similar to previous observations associated with celiac disease [3]. In the past, some have hypothesized that pharmacological or biological agents may have amplified or triggered the symptomatic presentation of a unrecognized celiac disease, however, evaluation of cases of olmesartan-associated enteropathy indicates that this is a distinct clinicopathologic entity that may follow prolonged and chronic drug treatment.

\section{Angiotensin II Receptor Antagonists}

Olmesartan is only one chemical formulation of a structurally related family of pharmacologic agents, or sartans, designed to block the activation of angiotensin II AT1-receptors and, as a result, modulate the reninangiotensin system. These drugs directly block AT1 receptors causing vasodilation, reduced secretion of vasopressin and reduced secretion of aldosterone leading to a reduction in blood pressure. Most sartans, including olmesartan, have tetrazole (a ring with 4 nitrogen and 1 carbon) and imidazole groups.

\section{Olmesartan and Diarrhea}

Olmesartan was first approved in April 2002 by the FDA for treatment of hypertension [4]. This drug has a biological half-life of 14 to 16 hours and is primarily cleared by the liver and kidneys. The usual daily dosage range is 10 to $40 \mathrm{mg}$. In earlier pre-clinical studies, adverse events, specifically diarrhea associated with olmesartan, were similar to placebo [4] with no reports of sprue-like intestinal disease. Subsequently, symptoms including lateonset diarrhea and weight loss related to olmesartan were noted by the FDA through its Adverse Event Reporting System. Patients apparently improved clinically after cessation of olmesartan and, in some, re-challenge caused a positive result [4]. In 2012, reports first appeared providing evidence of a sprue-like enteropathy attributed to omesartan in 2 case reports and a case series of 22 patients [5,6,7] followed by an FDA label change in 2013 [4].

\section{Initial Reports}

Investigations in a referred patient series [6] revealed that most were continuously treated with 10 to $40 \mathrm{mg}$ daily of olmesartan for varying periods of several months 
to years. Subsequent case studies from other centers have confirmed most of these initial descriptive features. Most have been female, mainly Caucasian, with ages in the initial case series ranging from 47 to 81 years (median 69.5 years). Many were reported to be obese or overweight before the development of diarrhea and subsequent weight loss. Chronic and persistent diarrhea with small intestinal mucosal inflammatory changes were evident, in some, initially attributed to celiac disease. However, a response to a gluten-free diet was not evident. Some had been labeled with non-responsive or refractory celiac disease, and others with collagenous sprue, and so were referred because of persistent symptoms [6]. In many, symptoms were severe, necessitating hospitalization with 14 patients suffering from marked dehydration and 4 requiring treatment for acute renal failure. Other symptoms included nausea, vomiting and abdominal pain. Subsequent studies included negative celiac serologic studies (eg., tissue transglutaminase antibodies). HLA-DQ typing was completed in 21 patients with DQ2 present in 15 and DQ8 in 2 patients. A total of 4 patients were negative for DQ2 and DQ8. Anti-enterocyte antibodies were negative in 16 of 19 patients tested. Anemia and hypoalbuminemia were frequent and multiple electrolyte deficiencies were common. Zinc deficiency was also detected in 7 patients. In some, gastric and colonic mucosal inflammatory changes were noted, including some with focal collagen deposition. Cessation of olmesartan treatment, however, led to a dramatic clinical improvement including weight gain and normalization of small intestinal histopathological changes. Interestingly, in an earlier report from 2010 from the same clinic, chronic olmesartan use had already been recorded in 8 of 30 patients with an enteropathy thought to pathologically have some resemblance to collagenous sprue [8], but a direct causative linkage with olmesartan per se had not been recognized. Like the histopathological changes in untreated celiac disease, the changes in collagenous sprue appear to reflect heterogenous causes [9], including medications, like olmesartan. It is also noteworthy that a further asymptomatic patient was also described in 2012 from the same clinic by a separate author raising the possibility of a wider clinical and histopathological spectrum of changes with olmesartan use [9].

\section{Pathogenesis}

The pathogenesis of this entity still requires definition. A pro-drug, olmesartan medoxomil is known to be metabolized by drug metabolizing enzymes, specifically esterase activities, in the intestinal tract and liver to the active agent, olmesartan, but its subsequent role in development of the enteropathy has not been precisely defined.

Interestingly, increased bacterial counts were reported in the small intestine of patients with olmesartan enteropathy, but antibiotic treatment reportedly did not provide symptomatic improvement. Nevertheless, these findings may be important because an altered luminal microbiome may be relevant in the development of many intestinal disorders, including other possible immune-mediated intestinal disorders, such as olmesartan enteropathy.

Indeed, the apparently long duration of drug treatment before clinically severe disease developed initially suggested that an immediate dose-related or direct toxic mechanism of injury could be reasonably excluded and may have been a factor in actually making initial recognition of this new form of drug-induced enteropathy difficult. Moreover, even if a specific immune-mediated mechanism of injury were eventually demonstrated, a type 1 hypersensitivity reaction would be less likely. Instead, a novel mechanism, including a yet-to-be-defined cell-mediated immune mechanism could occur [9].

Some have argued that the sero-detection of antienterocyte antibodies in some patients with olmesartan enteropathy is indicative of an autoimmune pathogenesis, or may even mimic some features of an autoimmune enteropathy [10]. However, not all patients tested to date with olmesartan enteropathy have detectable antienterocyte antibodies.

Some older investigations in mammalian kidney suggested that angiotensin receptor blockade may inhibit transforming growth factor-beta, a possible regulatory element in gut immune homeostasis [11]. As a result, some believe that either the oral prodrug or an active metabolite may be involved in causing this intestinal disorder. Additional studies are still needed to clarify the regulatory effects of this growth factor and other immune mediators in the intestinal mucosa, and changes with different pharmacological agents, including olmesartan. Indeed, in a recent study, the olmesartan acid portion of olmesartan medoxomil was associated with increased intestinal cell expression of the mediator IL-15 thought to cause disruption of the tight junction protein ZO-1 [12]. The authors hypothesized that those patients developing olmesartan enteropathy may be unable to down-regulate IL-15 expression.

In another very recent clinical report [13], two cases of olmesartan enteropathy were reported with the notation that an unidentified infectious gastrointestinal disorder seemed to function as a precipitant trigger for the severe malabsorption during the course of the natural history of this disorder.

The extent of this unusual enteropathy compared to other sartans is unknown, but these agents differ many fold in terms of measureable pharmacokinetic properties including pressor inhibition, AT1 affinity and biological half -life. To date, only 2 other sartans have only been very rarely reported to cause a similar drug-induced entity, valsartan [14] and irbesartan [15].

In a recent study exploring the histopathological changes associated with olmesartan compared to other angiotensin receptor blockers, a wide spectrum of spruelike histopathologic changes in duodenal biopsies was identified [16], but the extent of these histopathologic changes in the rest of the small intestine and their correlation with the severity of the clinical illness were not studied.

\section{Natural History}

The natural history of this disorder remains of particular interest. If unrecognized, a severe enteropathy may lead to hospitalization for management of diarrhea, profound fluid depletion and malabsorption with multiple nutritional deficits. Some may also have a microscopic form of colitis and an olmesartan sprue-like enteropathy has also been associated with colonic perforation that responded to conservative measures alone [17]. With drug cessation, most reported 
patients, even those from larger referral experiences with severe symptoms, appear to improve with resolution of diarrhea, weight gain and normalization of mucosal inflammatory changes, including those that have the pathological features reminiscent of celiac disease or collagenous sprue [7,18]. Others have occasionally reported response only after further drug therapy including immune suppressants or a biological agent, suggesting an ongoing immune disorder in some that requires regulation [10]. In some [19,20], re-challenge studies were positive suggesting that an underlying or ongoing abnormality in mucosal immune regulation may be present. Further clinical studies, particularly in new-onset patients with short compared to long duration of symptoms may be useful.

\section{Frequency of Occurrence}

This has been difficult to assess because of limited data, largely from referral centers. Even since the first published reports of olmesartan enteropathy, there has been some controversy regarding the degree of severity of the clinical presentation from severe clinical symptoms with marked histopathological changes to asymptomatic disease [6,7]. In part, controversy may have reflected the novel nature of this entity, often complicated by initial misdiagnosis, including failed treatments with gluten-free diets or other therapies for prolonged periods, further mislabelling as refractory celiac disease or autoimmune enteropathy [10] and eventual referral to other treatment centers.

Clinical trial data with olmesartan suggested that the occurrence of diarrhea was no different from placebotreated participants. Indeed, a recent case-control study of patients treated with angiotensin receptor blockers, including olmesartan, and referred for endoscopic studies to explore the cause of diarrhea compared to non-diarrhea controls showed no difference leading the investigators to conclude that olmesartan enteropathy must be a rare adverse event [21]. In contrast, a French nationwide observational cohort study of over 4 million patients noted a significant increase in discharge diagnosis of malabsorption in olmesartan users compared to users of ACE inhibitors [22]. Interestingly, statistical differences only appeared after at least a year of exposure to the olmesartin paralleling the clinical observation related to the need for extended drug exposure before development of malabsoprtion.

\section{Conclusion}

Olmesartan may cause a severe enteropathy with diarrhea, malabsorption and weight loss. Small intestinal histopathologic changes may resemble celiac disease and, in some, collagenous sprue. Both gastric and colonic microscopic changes may occur. Like other drug-induced enteropathies, complete resolution of the clinical features as well as the histopathologic changes may occur with cessation of the drug.

Clinicians need to be wary of this novel entity to avoid ongoing patient morbidity associated with continued use of olmesartan and incorrect use of other powerful immunosuppressive agents to treat diagnoses that may have similar clinicopathological features to olmesartan enteropathy. Moreover, the similarities of this entity to other common intestinal disorders, including celiac disease, may be useful to further investigate their complex immunological and histopathologic features.

\section{References}

[1] Freeman HJ. Refractory celiac disease and sprue-like intestinal disease. World J Gastroenterol 2008; 14: 828-830.

[2] Freeman HJ. Drug-induced sprue-like intestinal disease. International J Celiac Dis 2014; 2: 49-53.

[3] Freeman HJ. Collagenous mucosal inflammatory diseases of the gastrointestinal tract. Gastroenterology 2005; 129: 138-350.

[4] Tran TH, Li H. Olmesartan and drug-induced enteropathy. P \& T 2014; 39: 47-50.

[5] de Fonseka A, Tuskey A, Moskalul C. A case of olmesartaninduced enteropathy. Inflamm Bowel Dis 2012; 18: S17.

[6] Rubio-Tapia A, Hermann ML, Ludvigsson JF, Kelly DG, Mangan TF, Wu TT, Murray JA. Severe spruelike enteropathy associated with olmesartan. Mayo Clin Proc 2012; 87: 732-738.

[7] Talbot GH. Small bowel histopathologic findings suggestive of celiac disease in an asymptomatic patient receiving olmesartan. Mayo Clin Proc 2012; 87: 1231-1232.

[8] Rubio-Tapia A, Talley NJ, Gurudu SR, Wu TT, Murray JA. Gluten-free diet and steroid treatment are effective therapy for most patients with collagenous sprue. Clin Gastrointest Hepatol 2010; 8: 344-349.

[9] Freeman HJ. Collagenous sprue. Canad J Gastroenterol 2011; 25: 189-192.

[10] Scialom S, Malamut G, Meresse B, Guegan N, Brousse N, Verkarre V, Derrieux C, Macintyre E, Seksik P, Savoye G, Cadiot G, Vuitton L, Marthey L, Carbonnel F, Cerf-Bensussan N, Cellier C. Gastrointestinal disorder associated with omesartan mimics autoimmune enteropathy. PLoS One 2015; 10: e0125024.

[11] Kagami S, Border WA, Miller DE, Noble NA. Angiotensin II stimulates extracellular matrix protein synthesis through induction of transforming growth factor-beta expression in rat glomerular mesangial cells. J Clin Invest 1994; 93: 2431-2437.

[12] Narietta EV, Nadeau AM, Cartee AK, Singh I, Rishi A, Choung RS, Wu TT, Rubio-Tapia A, Murray JA. Immunopathogenesis of olmesartan-associated enteropathy. Aliment Pharmacol Ther 2015; 42: 1303-1314.

[13] Schiepatti A, Biagi E, Cumetti D, Luinetti O, Sonzogni A, Mugellini A, Corrazza GR. Olmesartan-associated enteropathy: new insights on the natural history? Scand J Gastroenterol 2016; 51: 152-156.

[14] Herman ML, Rubio-Tapia A, Wu TT, Murray JA. A case of severe sprue-like enteropathy associated with valsartan. ACG Case Rep J 2015; 2: 92-94.

[15] Marietta EV, Cartee A, Rishi A, Murray JA. Drug-induced enteropathy. Dig Dis 2015; 33: 215-220.

[16] Lagana SM, Braunstein ED, Arguelles-Grande C, Bhagat G, Green PHk, Lebwohl B. Sprue-like histology in patients with abdominal pain taking olmesartan compared with other angiotensin receptor blockers. J Clin Pathol 2015; 68: 29-32.

[17] Abdelghany M, Gonzales L $3^{\text {rd }}$, Slater J, Begley C. Olmesartan associated sprue-like enteropathy and colon perforation. Case Rep Gastrointest Med 2014; 2014: 494098

[18] Nielsen JA, Steephen A, Lewin M. Angiotensin-II inhibitor (olmesartan)-induced collagenous sprue with resolution following discontinuation of drug. World J Gastroenterol 2013; 19: 69286930.

[19] Theophile H, David XR, Miremont-Salame G, Harambukru F. Five cases of sprue-like enteropathy in patients treated by olmesartan. Dig Liver Dis 2014; 46: 465-469.

[20] Marthey L, Cadiot G, Seksik P, Pouderoux P, Lacroute J, Skinazi F, Mesnard B, Chayvialie JA, Savoye G, Druez A, Parlier D, Abitbol V, Gompel M, Eoche M, Poncin E, Bobichon R, Colardelle P, Wils P, Salloum H, Peschard S, Zerbib F, Meresse B, Cerf-Bensussan N, Malamut G, Carbonnel F. Omesartanassociated enteropathy: results of a national survey. Aliment Pharmacol Ther 2014; 40: 1103-1109.

[21] Greywoode R, Braunstein ED, Arguelles-Grande C, Green PH, Lebwohl B. Olmesartan, other antihypertensives, and chronic diarrhea among patients undergoing endoscopic procedures: a case-control study. Mayo Clin Proc 2014; 89: 1239-1243. 